\title{
Experimental evidence for real-time song frequency shift in response to urban noise in a passerine bird
}

\author{
Eira Bermúdez-Cuamatzin 1 , \\ Alejandro A. Ríos-Chelén ${ }^{1, *}$, Diego Gil ${ }^{2}$ \\ and Constantino Macías Garcia ${ }^{1}$
}

${ }^{1}$ Departamento de Ecología Evolutiva, Instituto de Ecologia, Universidad Nacional Autónoma de México, A.P. 70-275, C.P. 04510 México, D.F, México

${ }^{2}$ Departamento de Ecología Evolutiva, Museo Nacional de Ciencias Naturales (CSIC), fosé Gutiérrez Abascal 2, 28006 Madrid, Spain *Author for correspondence (aarios@ecologia.unam.mx).

Research has shown that bird songs are modified in different ways to deal with urban noise and promote signal transmission through noisy environments. Urban noise is composed of low frequencies, thus the observation that songs have a higher minimum frequency in noisy places suggests this is a way of avoiding noise masking. Most studies are correlative and there is as yet little experimental evidence that this is a short-term mechanism owing to individual plasticity. Here we experimentally test if house finches (Carpodacus mexicanus) can modulate the minimum frequency of their songs in response to different noise levels. We exposed singing males to three continuous treatments: low-high-low noise levels. We found a significant increase in minimum frequency from low to high and a decrement from high to low treatments. We also found that this was mostly achieved by modifying the frequency of the same low-frequency syllable types used in the different treatments. When different low-frequency syllables were used, those sung during the noisy condition were longer than the ones sang during the quiet condition. We conclude that house finches modify their songs in several ways in response to urban noise, thus providing evidence of a short-term acoustic adaptation.

Keywords: house finch; Carpodacus mexicanus; birdsong; urban noise; acoustic adaptation

\section{INTRODUCTION}

In acoustic communication, noise can be defined as any sound that reduces the ability of a receptor to detect acoustic signals [1]. Noise is ubiquitous in nature; however, human activities have increased the amount of noise that animals are exposed to, and evidence suggests that urban noise is a selective pressure that has shaped the song of birds inhabiting sound-polluted environments (reviews in $[1,2]$ ).

Selection has promoted several mechanisms that enhance the effectiveness of signal detection under

noisy conditions. A first mechanism, the Lombard effect, is characterized by an increase in sound amplitude as a response to noise [3-5]. Additionally, birds can increase the redundancy of signals so that the message has an increased chance of getting across in noisy environments [6], or sing at times when noise level is lower [7]. Another possibility is to sing higher pitched songs to reduce their acoustic overlap with low-frequency noise [8-11]. A recent study has shown that urban populations of great tits (Parus major) have an increased minimum frequency in comparison to the countryside populations, and that this modification is best explained as a response to noise perturbation [12]. However, there is still little evidence disentangling the relative contribution of natural selection, differential learning and individual plasticity in generating this noise-dependent change in frequency (but see [13] for a case of cultural evolution). In a previous correlative study in the house finch (Carpodacus mexicanus), we found that males modulated the frequency of their song by increasing the minimum frequency of certain syllable types, when bursts of urban noise occurred during the recording session, suggesting that immediate, real-time responses are possible in this species [8]. In the great tit, it has been shown that when confronted with low-frequency urban noise, males sing, for longer times, song types with higher low-frequency notes [14].

Here we set out to experimentally test the hypothesis that house finches can plastically modify the frequency of their songs, by exposing singing males with different levels of urban noise in controlled captivity conditions.

\section{MATERIAL AND METHODS}

We obtained birds from authorized local suppliers in Mexico City, which had been captured as adults in different areas of Mexico, including Mexico City and San Luis Potosí. Birds were kept in individual cages, and fed with an ad libitum combination of seed mix and fruit. Light/dark cycle was kept at $12: 12$. Before the experiment, all males were implanted with testosterone to induce singing. Twenty one males were used in this experiment.

The experiment was run in March-April 2009, in the mornings 8.00-14.00 and afternoons 16.00-18.00. Individually caged males were placed outdoors in an arena set in a quiet open area of the Universidad Nacional Autónoma de México (UNAM) campus. Males were flanked at approximately $10 \mathrm{~cm}$ by two individually caged females to induce singing. Males were visually separated from females, but could interact vocally. Four females were changed between males to avoid habituation. This set of three cages was presented with a loudspeaker (Mineroff SME-AFS, frequency response: $100 \mathrm{~Hz}-12 \mathrm{kHz}$ ) from which the acoustic stimuli were broadcast using a laptop (HP Pavilion dv1000), while the experimenter was hidden from the birds at a distance of $3.5 \mathrm{~m}$ from the arena (figure 1).

All males were sequentially exposed to two different sound stimuli: low (44-57 dB) and high (56-65 dB; see below) levels of urban noise, in a 'sandwich' fashion of playbacks: low-high-low. Each bird received a different noise recording to avoid pseudoreplication. Treatments were prepared from atmospheric recordings at a busy avenue near UNAM, Mexico City, with a microphone Sennheiser ME66 connected to a Marantz PMD221 tape recorder, using TDK SA60 tapes. From a $2 \mathrm{~h}$ recording, $30 \mathrm{~s}$ segments of noise were randomly selected, normalized to the same amplitude and prepared using Avisoft SASLAB PRO v. 2.0. Since sound pressure was not even through any given segment, noise level during each playback was not a fixed value, but rather a range, which we kept at the values indicated above.

The experimental procedure started by taking the females from their home cages and placing them in the flanking cages of the arena, where they were left to habituate for $15 \mathrm{~min}$. Then a male was taken from his cage and placed in the experimental cage. A continuous recording began once he started singing. After it had produced five songs under ambient noise (31-39 dB), playbacks of 


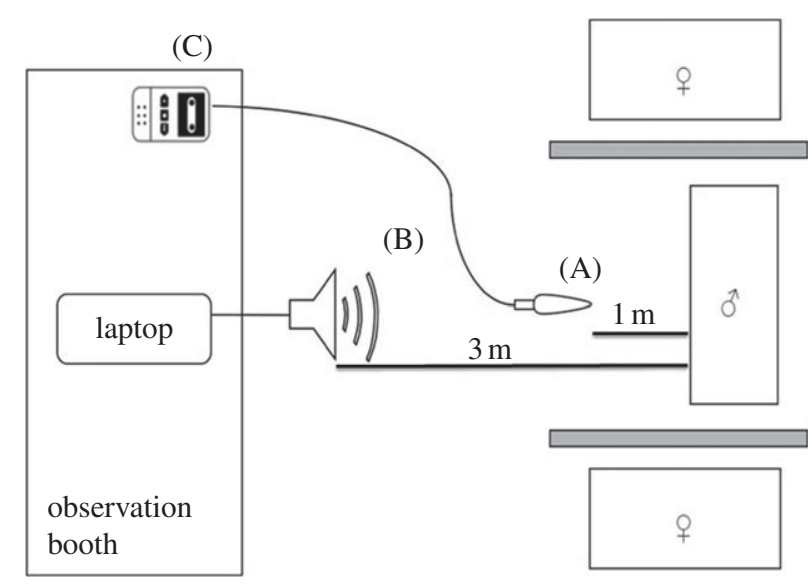

Figure 1. Song recording arena. The cages of experimental individuals are represented by rectangles on the right. The divisions between cages are observed in grey. The microphone (A) is shown connected by a cable to the recorder (C). The loudspeaker (B) is shown connected to a laptop.

noise stimuli were broadcasted until we obtained a minimum of three songs under each of low, high and low noise conditions. The recordings of the songs were made with a Sennheiser ME66 directional microphone connected to a Marantz digital recorder PMD660.

Noise intensity was measured with a digital sound pressure meter (SEW 2310 SL, ANSI S1.4 Type II), with a frequency range of $30-130 \mathrm{~dB}$, A-weight mode, and slow-response setting.

Of a total of 21 males, 10 males sang during the first (low) and second (high) playbacks, and only five continued singing during the third playback (low). As data were normally distributed, we used a paired-t-test to compare the change from low to high in those 10 males, and a Wilcoxon matched-pairs test to compare the songs of those five males that kept on singing from high to low.

We measured song length, minimum and maximum frequencies of songs, and length of each syllable within songs. Additionally, we made a syllable catalogue to analyse whether birds used the same or different minimum-frequency syllable types in different treatments. These minimum-frequency syllable types were classified as shared or non-shared, depending on whether or not they were used by an individual in the different treatments.

\section{RESULTS}

We found a significant increase in minimum frequency from low to high $(t=-3.71$, d.f. $=9, p=0.005$; figure 2). Neither song duration $(t=1.30$, d.f. $=9$, $p=0.22)$ nor maximum song frequency $(t=0.17$, d.f. $=9, p=0.86)$ changed between treatments. The mean length of syllables within songs did not differ between treatments $(t=0.21$, d.f. $=9, p=0.83)$. In the sample of males that also sang in the third playback (low), we found a significant decrease in minimum frequency from high to low $(Z=-2.023, n=5$, $p=0.04$; figure 2). As expected, noise had a strong low-frequency component (peak frequency, mean \pm s.e. $=231 \pm 28 \mathrm{~Hz}$, range $=184-475 \mathrm{~Hz}$ ).

Males sang both shared- and non-shared-syllable types during noise exposure (table 1). We found a strong significant increase in song frequency when we analysed only the songs with shared-syllable types $(t=-5.587$, d.f. $=9, p<0.001$, see the electronic supplementary material), but the effect fell short of significance when we analysed those with nonshared-syllable types $(t=-2.035$, d.f. $=7, p=0.08)$. We found that the songs with shared syllables were shorter during noise exposure $(t=2.531$, d.f. $=9$, $p=0.03)$. This was not the case for songs with

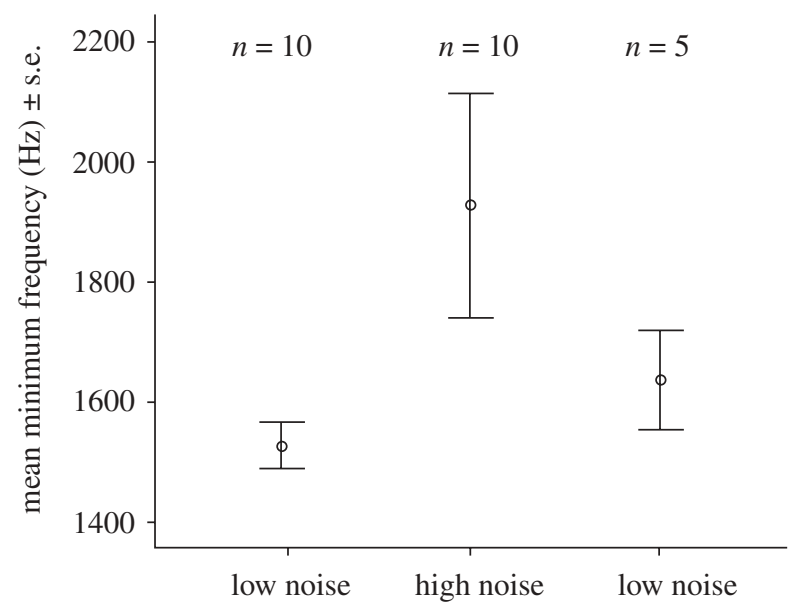

Figure 2. Mean minimum frequency in the three treatments. Males increased the minimum frequency of their songs from low to high and then decreased it from high to low. See text for statistics.

Table 1. Percentages of shared-syllable types and shared syllables among songs produced by males during treatments. Since the percentage of shared-syllable types and shared syllables is not $100 \%$, this means that the songs produced in different treatments had non-shared-syllable types also.

\begin{tabular}{lll}
\hline male & $\%$ shared-syllable types & $\%$ shared syllables \\
\hline 1 & 33.3 & 60.0 \\
2 & 60.0 & 83.3 \\
3 & 12.5 & 30.0 \\
4 & 22.2 & 35.7 \\
5 & 50.0 & 83.3 \\
6 & 20.0 & 47.0 \\
7 & 16.6 & 25.0 \\
8 & 14.2 & 41.6 \\
9 & 16.6 & 64.2 \\
10 & 33.3 & 55.5 \\
\hline
\end{tabular}

non-shared syllables $(t=0.858$, d.f. $=7, p=0.41)$. Finally, non-shared-syllable types were longer than the syllables they replaced $(t=-2.605$, d.f. $=7$, $p=0.03)$; whereas shared-syllable length did not vary between treatments $(t=1.435$, d.f. $=9, p=0.18)$.

\section{DISCUSSION}

Our experiment shows that house finch males shift the minimum frequency of their songs in response to an increase in the amplitude of urban noise. This dynamic change is indicative of real-time plasticity, as suggested by correlative data in the same species [8].

Frequency shifts as a response to urban noise have been found in several bird species, and evidence suggests that it is a widespread strategy used by oscines to increase the salience of their songs in noisy urban environments $[8-10,12,15]$, leading to interpopulation song divergence [16].

Song styles are markedly different among species, and thus it is not surprising that those species that achieve this shift differ in the mechanisms used to accomplish it. In the great tit, song frequency differences between noisy and quiet populations seem to 
be owing to differential use of song-types, so that in noisy situations birds may favour the use of highpitched songs [12]. In the house finch, a species with more versatile songs, we have found that the same syllable types are used in noisy and quiet areas, and that birds can modify the minimum frequency of a given syllable type depending on the noise level [8, this study], but that males also use other novel minimum syllable types (this study). The fact that the frequency shift was stronger when we considered only those songs with shared syllables suggests that the frequency shift is achieved mostly by increasing the pitch of sharedsyllable types, which is in accordance with previous correlative findings [8]. A study in the Bengalese finch (Lonchura striata) has shown that birds are able to increase or decrease the frequency of a given syllable in response to aversive conditioning, suggesting that minor variations in frequency of a given note can be perceived and controlled [17].

Here we also show that house finches' songs can be classified into two distinct groups in relation to their response to noise; those with shared-syllable types and those with non-shared-syllable types. The former have a stronger effect in frequency shifts and are shortened during noise exposure. In contrast, non-sharedsyllable types, whose role in frequency shifts is at best marginal, are elongated during noise exposure. Thus house finches have at their disposal two strategies to deal with urban noise: frequency increase, and temporal elongation. A similar temporal change in vocalization elements have been previously reported by Brumm et al. [18] in the marmoset (Callithrix jacchus). It remains to be studied whether the ability to shift song frequency in response to noise is a widespread passerine ability or whether it is limited to those species that learn their songs.

Authorization to collect birds and to conduct this research was granted by the Mexican Ministry for the Environment (SEMARNAT).

We thank Isabel López, Edgar Ávila, Ivan Delgado, Ruth Tejeda, Maricela López, Marcela Méndez and SEMARNAT for a licence to collect birds. This project was financed by a research grant from Fundación BBVA to DG and CMG. EBC was supported with a $\mathrm{PhD}$ scholarship from CONACYT. We thank two anonymous referees for constructive and useful comments.

1 Brumm, H. \& Slabbekoorn, H. 2005 Acoustic communications in noise. Adv. Study Behav. 35, 151-209. (doi:10.1016/S0065-3454(05)35004-2)

2 Patricelli, L. \& Blickley, J. 2006 Avian communication in urban noise: causes and consequences of vocal adjustment. Auk 123, 639-649. (doi:10.1642/00048038(2006)123[639:ACIUNC]2.0.CO;2)
3 Brumm, H. \& Todt, D. 2002 Noise-dependent song amplitude regulation in a territorial songbird. Anim. Behav. 63, 891-897. (doi:10.1006/anbe.2001.1968)

4 Cynx, J., Lewis, R., Tavel, B. \& Tse, H. 1998 Amplitude regulation of vocalizations in noise by a songbird, Taeniopygia guttata. Anim. Behav. 56, 107-113. (doi:10. 1006/anbe.1998.0746)

5 Kobayashi, K. \& Okanoya, K. 2003 Context-dependent song amplitude control in Bengalese finches. Neuro. Rep. 14, 521-524.

6 Brumm, H. \& Slater, P. J. B. 2006 Ambient noise, motor fatigue, and serial redundancy in chaffinch song. Behav. Ecol. Sociobiol. 60, 475-481. (doi:10.1007/ s00265-006-0188-y)

7 Fuller, R. A., Warren, P. H. \& Gaston, K. J. 2007 Daytime noise predicts nocturnal singing in urban robins. Biol. Lett. 3, 368-370. (doi:10.1098/rsbl.2007.0134)

8 Bermúdez-Cuamatzin, E., Ríos-Chelén, A. A., Gil, D. \& Macías Garcia, C. 2009 Strategies of song adaptation to urban noise in the house finch: syllable pitch plasticity or differential syllable use? Behaviour 146, 1269-1286. (doi:10.1163/156853909X423104)

9 Fernández-Juricic, E., Poston, R., De Collibus, C., Morgan, T., Bastain, B., Martin, C., Jones, K. \& Treminio, R. 2005 Microhabitat selection and singing behavior patterns of male house finches (Carpodacus mexicanus) in urban parks in a heavily urbanized landscape in the western U. S. Urban Habitats 3, 49-69.

10 Slabbekoorn, H. \& Peet, M. 2003 Birds sing at higher pitch in urban noise. Nature 426, 267-267. (doi:10. $1038 / 424267 a)$

11 Slabbekoorn, H. \& Smith, T. B. 2002 Habitat dependent song divergence in the little greenbul: an analysis of environmental selection pressures on acoustic signals. Evolution 56, 1849-1858. (doi:(10.1554/00143820(2002)056[1849:HDSDIT]2.0.CO;2)

12 Slabbekoorn, H. \& den Boer-Visser, A. 2006 Cities change the songs of birds. Curr. Biol. 16, 2326-2331.

13 Luther, D. \& Baptista, L. 2010 Urban noise and the cultural evolution of bird songs. Proc. R. Soc. B 277, 469-473. (doi:10.1098/rspb.2009.1571)

14 Halfwerk, W. \& Slabbekoorn, H. 2009 A behavioural mechanism explaining noise-dependent frequency use in urban birdsong. Anim. Behav. 78, 1301-1307. (doi:10.1016/j.anbehav.2009.09.015)

15 Nemeth, E. \& Brumm, H. 2009 Blackbirds sing higherpitched songs in cities: adaptation to habitat acoustics or side-effect of urbanization? Anim. Behav. 78, 637-641. (doi:10.1016/j.anbehav.2009.06.016)

16 Ripmeester, E. A. P., Kok, J. S., van Rijssel, J. C. \& Slabbekoorn, H. 2010 Habitat-related birdsong divergence: a multi-level study on the influence of territory density and ambient noise in European blackbirds. Behav. Ecol. Sociobiol. 64, 409-418.

17 Tumer, C. \& Brainard, M. 2007 Performance variability enables adaptive plasticity of 'crystallized' adult birdsong. Nature 450, 1240-1244. (doi:10.1038/nature06390)

18 Brumm, H., Voss, K., Köllmer, I. \& Todt, D. 2004 Acoustic communication in noise: regulation of call characteristics in a New World monkey. F. Exp. Biol. 207, 443-448. (doi:10.1242/jeb.00768) 